\title{
The role of lumbar puncture in children with suspected central nervous system infection Rachel Kneen ${ }^{1}$, Tom Solomon*2 and Richard Appleton ${ }^{1}$
}

Address: ${ }^{1}$ The Roald Dahl EEG Unit, Royal Liverpool Children's NHS Trust, Alder Hey, Liverpool, L12 2AP, UK and ${ }^{2}$ Department of Neurological Science, University of Liverpool, Walton Centre for Neurology and Neurosurgery, Liverpool, UK and Department of Medical Microbiology and School of Tropical Medicine, University of Liverpool, Liverpool, UK

E-mail: Rachel Kneen - rachel.kneen@btopenworld.com; Tom Solomon* - tsolomon@liv.ac.uk; Richard Appleton - Richard.Appleton@RLCHTR.NWEST.NHS.UK

${ }^{*}$ Corresponding author

Published: 2 September 2002

BMC Pediatrics 2002, 2:8
Received: 20 May 2002

Accepted: 2 September 2002

This article is available from: http://www.biomedcentral.com//47I-243I/2/8

(C) 2002 Kneen et al; licensee BioMed Central Ltd. This article is published in Open Access: verbatim copying and redistribution of this article are permitted in all media for any non-commercial purpose, provided this notice is preserved along with the article's original URL.

\begin{abstract}
Background: The use of the lumbar puncture in the diagnosis of central nervous system infection in acutely ill children is controversial. Recommendations have been published but it is unclear whether they are being followed.

Methods: The medical case notes of 415 acute medical admissions in a children's hospital were examined to identify children with suspected central nervous system infection and suspected meningococcal septicaemia. We determined whether lumbar punctures were indicated or contraindicated, whether they had been performed, and whether the results contributed to the patients' management.
\end{abstract}

Results: Fifty-two children with suspected central nervous system infections, and 43 with suspected meningococcal septicaemia were identified. No lumbar punctures were performed in patients with contraindications, but only $25(53 \%)$ of 47 children with suspected central nervous system infection and no contraindications received a lumbar puncture. Lumbar puncture findings contributed to the management in $18(72 \%)$ of these patients, by identifying a causative organism or excluding bacterial meningitis.

Conclusion: The recommendations for undertaking lumbar punctures in children with suspected central nervous system infection are not being followed because many children that should receive lumbar punctures are not getting them. When they are performed, lumbar puncture findings make a useful contribution to the patients' management.

\section{Background}

The use of the lumbar puncture (LP) in the diagnosis of central nervous system (CNS) infection in children is controversial [1-3]. In the UK, the use of the LP in CNS infections has declined dramatically since the 1960 's, when it was considered an essential investigation for such patients
[4]. LP use began to decline after concerns were expressed that they may be precipitating brainstem herniation and death in some patients [5-7]. Although the causal association between LP and cerebral herniation remains unproven, recommendations were published as to which patients should and should not receive a LP $[1,2,8]$. How- 
ever, the role of the LP has been questioned again recently because of the suggestion that, since the arrival of newer diagnostic techniques - especially the polymerase chain reaction (PCR), the LP now contributes little to patient management [9]. The purpose of this study was to determine whether the recommendations for LP are being followed, and whether the CSF findings obtained contributed to patients' management. We show that only $53 \%$ of patients that should have received an LP had one, yet in nearly three quarters of these patients it helped in the management.

\section{Methods}

Case notes of children admitted to this paediatric secondary and tertiary referral hospital from January $1^{\text {st }}$ to April $30^{\text {th }} 2000$ were reviewed to see if a CNS infection or meningococcal septicamia were included in their differential diagnosis at admission. To identify such patients the notes of all patients with the following discharge diagnoses were looked at: acute respiratory, urinary, viral and meningococcal infection; febrile convulsions, tonsillitis, otitis media, meningitis or encephalitis, septicaemia and rash. Then it was determined whether there was clinical evidence to suspect CNS infection or meningococcal septicaemia. CNS infections were suspected in children with a febrile illness, and at least one of the following [10]: neck stiffness, bulging fontanelle, photophobia, severe headache (severe enough to require assessment in hospital), irritability, reduced level of consciousness, focal neurological signs or convulsions (excluding simple febrile convulsions) [11]. This included all sick infants less than six months of age who had no obvious focus for infection. Case notes of children with long-term medical problems were excluded.

Lumbar punctures were considered to have been indicated in patients with suspected CNS infection and with no contraindications (table 1$)[1,2,8,12,13]$. LPs were also considered to be contraindicated in children with meningococcal septicaemia but no evidence of CNS infection [3]. LP findings were considered to have contributed to patients' management if additional diagnostic microbiological information was obtained (above that obtained from other investigations), or if, by ruling out a diagnosis, antibiotic or antiviral drugs could be discontinued and earlier hospital discharge was possible.

\section{Results}

Four hundred and fifteen of 448 patients' case notes were examined (33 were unavailable). The median (range) age of the children was 14 months (nine days to 16 years). Ninety-five children with either suspected CNS infection or suspected meningococcal septicaemia were identified. Of these, sixty-eight ( $72 \%$ ) presented directly to the emergency department, $15(16 \%)$ were referred by general
Table I: Indications for LP in children with suspected CNS infections*

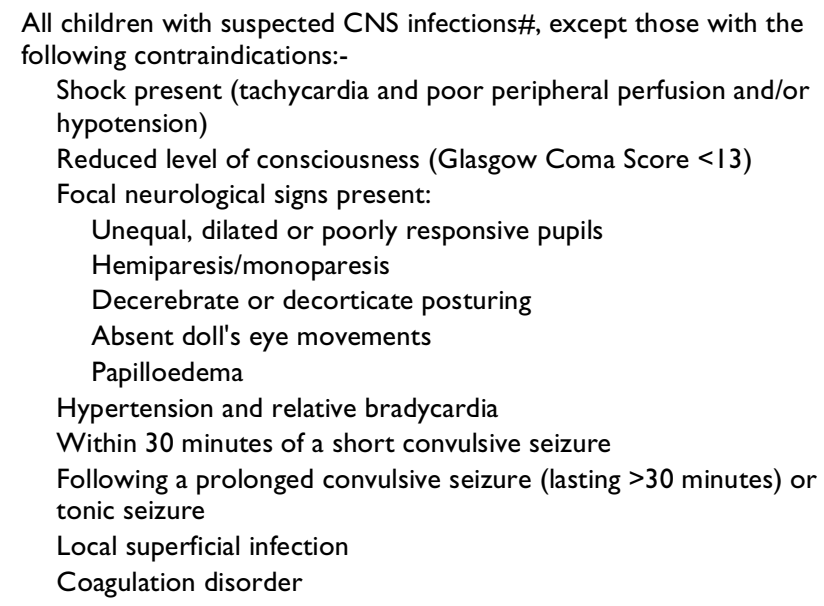

*Modified from references: $[1-3,8,12,13]$ \#Including infants $<6$ months old, as part of septic screen LP should be reconsidered, following initial treatment in those who have a contraindication at presentation

practitioners, and $12(12 \%)$ were transferred directly to the paediatric intensive care (PICU) from local district general hospitals. Fifty-two $(12.5 \%)$ of the 415 patients had suspected CNS infection: 22 with suspected meningitis and no rash, 13 with suspected meningitis and a meningococcal rash, and 17 infants who were non-specifically unwell and febrile (median [range] age 33 days [9 days-5 months]) (table 2). LP was contraindicated on admission in five of the 13 patients with suspected meningitis and a meningococcal rash. All five had shock and poor perfusion and two had a reduced Glasgow Coma Score (GCS $=6$ and 8). Of the remaining 47 patients with suspected CNS infection, and no contraindications, only 25 (53\%) had an LP performed, 21 on admission and four when the clinical condition of the patient had stabilised. CSF opening pressure was not recorded in any patient. Microscopy and culture were undertaken on all CSFs. Protein and glucose levels were measured in 21 of the 25 samples, but a simultaneous plasma glucose was measured in only 8 of these 21 patients. 43 patients had suspected meningococcal septicaemia without CNS involvement (including 8 in whom viral infection with petechiae was considered the most likely diagnosis). LPs were not performed in any of these 43 children. No patient in any group died or had sequelae.

CSF analysis was abnormal in seven of the 25 patients (28\%). CSF bacterial culture was positive in three of these patients, all with negative blood cultures (table 2). $N$. meningitidis was cultured from the blood of one of the seven patients, and three had aseptic meningitis. Sterile CSF 
Table 2: Clinical and microbiological details of 52 children with suspected CNS infection and 43 with meningococccal septicaemia

\begin{tabular}{|c|c|c|c|c|}
\hline \multirow[t]{2}{*}{ Initial Clinical Impression } & \multicolumn{2}{|r|}{ LP Performed } & \multicolumn{2}{|r|}{ LP Not performed } \\
\hline & $\begin{array}{l}\text { No. of } \\
\text { patients }\end{array}$ & CSF findings and microbiology results & $\begin{array}{l}\text { No. of } \\
\text { patients }\end{array}$ & Microbiology results \\
\hline \multirow[t]{4}{*}{ Suspected meningoencephalitis, no rash } & 14 & Pleocytosis, N. meningitidis in CSF [2]* & 8 & N. meningitidis in BC \& PCR [I] \\
\hline & & Pleocytosis, N. meningitidis in $\mathrm{BC}[\mathrm{I}]$ & & N. meningitidis by PCR $[\mathrm{I}]$ \\
\hline & & Pleocytosis, E. coli in CSF [I] & & \\
\hline & & Pleocytosis, cultures negative [2] & & \\
\hline \multirow{3}{*}{$\begin{array}{l}\text { Suspected meningitis with } \\
\text { meningococcal rash }\end{array}$} & 1 & Pleocytosis, cultures negative $[\mathrm{I}]$ & $12 \#$ & N. meningitidis in $\mathrm{BC}(6$, including $4 \mathrm{PCR}+)$ \\
\hline & & & & N. meningitidis by PCR [2] \\
\hline & & & & H. Influenzae in endotracheal aspirate [I] \\
\hline Septic screen in infants $<6$ months old & 10 & CSF normal & 7 & E. coli in $\mathrm{BC}[\mathrm{I}]$ \\
\hline \multirow{3}{*}{$\begin{array}{l}\text { Meningococcal septicaemia } \\
\text { (LP Contraindicated) }\end{array}$} & 0 & & 43 & N. meningitidis in $B C(7$, including 5 PCR +$)$ \\
\hline & & & & N. meningitidis by PCR $[10]$ \\
\hline & & & & N. meningitidis by antigen test only [3] \\
\hline
\end{tabular}

\footnotetext{
* Numbers of patients in brackets \# 5 patients had contraindications to LP, (all were shocked, and 2 had deep come): 2 with BC, and I with PCR positive for $N$ meningitidis $\mathrm{BC}=$ blood cultures, $\mathrm{CSF}=$ cerebrospinal fluid, $\mathrm{WBC}=$ white blood cells $\mathrm{PCR}$ was only performed on blood
}

cultures at 48 hours enabled 15 patients to have antibiotics discontinued, one of whom also had acyclovir discontinued. Thus $15(60 \%)$ of 25 patients that received a LP had antibiotics stopped early, compared with three of 22 patients that should have received and LP but did not do so $(\mathrm{P}<0.001)$. Of the 43 children with suspected meningococcal septicaemia, 20 (46\%) had microbiological evidence of $N$. meningitidis infection either from blood cultures or PCR on a blood sample.

\section{Discussion}

This retrospective case note review has suggested that the recommendations for undertaking $L P$ in cases of suspected childhood CNS infection are not being followed. Although no LPs were performed in patients who should not have had them, only $53 \%$ of those patients who should have received an LP actually got one. Furthermore, in only eight of the 25 patients (32\%) who had an LP was the CSF examined fully, including a CSF/plasma glucose ratio. Although the reasons for this are unclear, it may be the well-publicised guidelines relating to emergency management of meningococcal septicaemia $[3,9]$ have become more widely and inappropriately applied to any patient with suspected CNS infection, whether or not there is an accompanying rash [14]. In addition, because children with suspected CNS infection are usually treated with antibiotics and/or antiviral drugs pending CSF culture results, some physicians may believe that CSF findings do not contribute to patient management. However, in our study, LP findings identified the causative organism in three of the four patients with proven bacterial meningitis and excluded bacterial meningitis in a further $15 \mathrm{pa}-$ tients, allowing antibiotics to be discontinued and an earlier discharge from hospital. Therefore CSF analysis gave additional clinically useful information in 18 (72\%) of the 25 patients in whom it was performed. The role of CSF analysis in patients with meningism and a suspected meningococcal rash remains controversial $[3,9]$. In our study seven such patients did not have an LP, and blood cultures or PCR was positive in five. Whether the remaining two children genuinely had meningococcal meningitis is not known, but a LP would clearly have confirmed or refuted the diagnosis. The advantages of obtaining a microbiological diagnosis extend beyond individual patient management. Knowing the organism allows appropriate prophylaxis to be recommended for close contacts, and it allows the PHLS to determine whether a series of meningitis cases really is an outbreak due to a single organism, or a cluster of unrelated cases.

The reduction in the number of LPs being performed by junior doctors may have wide-reaching consequences. What was once considered to be a routine and relatively safe investigation now appears to be relatively rare in our setting. This is in contrast to other parts of the world, particularly the tropics, where LP is still considered an essential investigation $[15,16]$. Whereas 10 years ago most doctors in the UK learnt to do LPs as medical students or house officers, this does not appear to be the case now [14]. The recent trend towards ward-based and shift work, 
where-by juniors often do not follow up patients they admit, has meant they are less likely to see the benefits of investigating patients fully.

\section{Conclusions}

Our study has shown that the recommendations for undertaking LPs in children with suspected CNS infection are not being followed, because many children that should receive LPs are not getting them. There are clear individual patient-management, public health and health economic implications if the findings of this study are mirrored in other paediatric units.

\section{Competing interests}

None declared.

\section{Authors' Contributions}

RK and TS conceived the idea for the study. All three authors contributed to the design. RK collected the data and analysed it with the help of TS, under the supervision of RD.

All three authors contributed to the writing of the manuscript.

\section{Acknowledgments}

We thank our clinical colleagues for supporting this work and Pat McCarrick for help with chart retrieval.

\section{References}

I. Addy D: When not to do a lumbar puncture. Archives of Disease in Childhood 1987, 62:873-875

2. Mellor $D$ : The place of computed tomography and lumbar puncture in suspected meningitis. Archives of Disease in Childhood 1992, 67:1417-1419

3. Pollard AJ, Britto J, Nadel S, DeMunter C, Habibi P, Levin M: Emergency management of meningococcal disease. Archives of Disease in Childhood 1999, 80:290-296

4. Harper JR: Timing of lumbar puncture in severe childhood meningitis. British Medical Journal 1985, 29 1:65 I-652

5. Horwitz SJ, Boxerbaum B, O'Bell J: Cerebral herniation on bacterial meningitis in childhood. Annals of Neurology 1978, 7:524-528

6. Slack J: Deaths from Meningococcal infection in England and Wales in 1978. Journal of the Royal College of Physicians of London 1982, 16:40-44

7. Rennick G, Shann F, de Campo ]: Cerebral herniation during bacterial meningitis in children. British Medical Journal 1993, 306:953955

8. Klein JO, Feigin RD, McCracken GH Jr: Report of the Task Force on Diagnosis and Management of Meningitis. Psediatrics 1986, 78:959-982

9. Wylie PAL, Stevens D, Drake W III, Stuart J, Cartwright K: Epidemiological and clinical management of meningococcal disease in West Gloustershire: retrospective, population based study. British Medical Journal 1997, 3 I 5:774-779

10. Solomon T, Dung NM, Kneen R, et al: Seizures and raised intracranial pressure in Vietnamese patients with Japanese encephalitis. Brain 2002, I 25:1084-1093

II. Verity CM, Butler NR, Golding J: Febrile convulsions in a national cohort followed up from birth. I. Prevalence and recurrence in the first five years of life. British Medical Journal 1985, 290: I3071310

12. Advanced Life Support Group: Advanced Paediatric Life Support. London: BMJ publishing group 1997

13. Appleton R, Choonara I, Martland T, Philips B, Scott R, Whitehouse $W$ : The treatment of convulsive status epilepticus in chil- dren. The Status Epilepticus Working Group. Archives of Disease in Childhood 2000, 83:415-419

14. Kneen R, Solomon T, Appleton RA: The role of lumbar punctures in CNS infections - a disappearing skill? Arch Dis in Child 2002, $181-183$

15. White NJ: Lumbar puncture in cerebral malaria (letter). Lancet 1991, 338:640-64|

16. Solomon T, Kneen R: Neurological Presentations. In: Lecture Notes on Tropical Medicine (Edited by: Beeching N, Gill G, eds) Oxford: Blackwell Science 2002

\section{Pre-publication history}

The pre-publication history for this paper can be accessed here:

http://www.biomedcentral.com/1471-2431/2/8/prepub
Publish with BioMed Central and every scientist can read your work free of charge

"BioMedcentral will be the most significant development for disseminating the results of biomedical research in our lifetime." Paul Nurse, Director-General, Imperial Cancer Research Fund

Publish with BMC and your research papers will be:

- available free of charge to the entire biomedical community

- peer reviewed and published immediately upon acceptance

- cited in PubMed and archived on PubMed Central

- yours - you keep the copyright

Submit your manuscript here: BioMedcentral.com http://www.biomedcentral.com/manuscript/ editorial@biomedcentral.com 